\title{
Antioxidantes, Atividade Física e Estresse Oxidativo em Mulheres Idosas
}

\section{Antioxidants, Physical Activity and Oxidative Stress in Older Women}

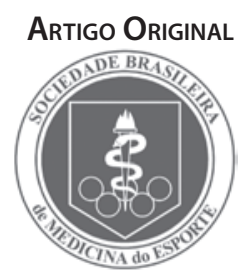

\author{
José Rubens Rebelatto ${ }^{1}$ \\ Rafael Jiménez ${ }^{2}$ \\ Marco A. Delgado 3 \\ Begoña Muguerza ${ }^{3}$ \\ Maria E. Muñoz ${ }^{2}$ \\ Ana I. Galan² \\ Roberto Méndez Sánchez ${ }^{4}$ \\ José Ignácio Calvo Arenillas ${ }^{4}$
}

1. Departamento de Fisioterapia da Universidade Federal de São Carlos, Brasil.

2. Departamento de Fisiologia e Farmacologia da Universidade de Salamanca, Espanha.

3. Departamento de I+D. Grupo Leche Pascual. Aranda de Duero, Burgos, Espanha.

4. Escola Universitária de Enfermagem e Fisioterapia da Universidade de Salamanca, Espanha.

\section{Endereço para correspondência:} Departamento de Fisioterapia, Universidade Federal de São Carlos, Rod. Washington Luiz Km 235, CEP 13565-905, São Carlos (SP) - Brasil.

Email: rubens@power.ufscar.br

Submetido em 12/072007

Versão final recebida em 19/10/2007 Aceito em 01/11/2007

\begin{abstract}
RESUMO
Objetivo: Verificar a influência da suplementação de vitaminas antioxidantes na dieta de mulheres idosas que praticam exercícios físicos regulares, sobre o estresse oxidativo, indicadores da saúde física e risco de enfermidades cardiovasculares (ECV). Método: Foram observados dois grupos (S e C) de mulheres com idades entre 60 e 80 anos participantes de um programa de atividades físicas durante 58 semanas, com freqüência de três vezes por semana e duração de 50 a 55 minutos cada sessão. A dieta habitual do Grupo $S(n=36)$ foi suplementada diariamente com $330 \mathrm{ml}$ de uma bebida antioxidante (Funciona ${ }^{\mathrm{T} M}$ ); o Grupo C ( $\mathrm{n=32}$ ) ingeriu água e se caracterizou como controle. Como indicadores do estresse oxidativo foram determinadas as concentrações plasmáticas de glutationa reduzida (GSH) e oxidada (GSSG), calculada a relação molar GSH/GSSG, e identificado o dano oxidativo em lipídios e proteínas. As condições físicas e cardiovasculares foram avaliadas por meio dos parâmetros antropométricos habituais (peso, altura e índice de massa corporal) e da pressão arterial. Resultados: O Grupo C apresentou aumentos significativos do estresse oxidativo, redução da pressão arterial e dos valores médios de indicadores de risco de ECV. O Grupo S teve o estresse oxidativo reduzido significativamente e apresentou incremento dos ganhos cardiovasculares. Não foram identificadas significâncias em relação aos efeitos ergogênicos. Conclusão: Os dados indicam que mulheres idosas que realizam exercícios físicos freqüentes melhoram suas condições físicas e cardiovasculares e que o suplemento dietético continuado de alimentos funcionais antioxidantes podem minimizar os efeitos danosos das espécies reativas de oxigênio.
\end{abstract}

Palavras-chave: atividade física, antioxidantes, estresse oxidativo, idosos, saúde cardiovascular.

\begin{abstract}
Objective: To verify the influence of dietary antioxidant supplementation in older women who regularly practice physical activities, on the occurrence of oxidative stress, physical health and risk of cardiovascular diseases (CVD). Method: Two groups ( $S$ and C) of women, with age ranging from 60 to 80 years old, were observed. Both groups took part in a physical activity program for 58 weeks, three times a week, for about 50 to 55 minutes each session. The diet of group $S(n=36)$ was daily supplemented with $330 \mathrm{ml}$ of a functional antioxidant beverage, Funciona ${ }^{\mathrm{TM}}$; Group C $(n=32)$ ingested water and was used as Control. As oxidative stress indicators, the plasmatic concentrations of reduced (GSH) and oxidized (GSSG) glutathione were determined; the molar GSH/GSSG ratio was calculated, and the oxidative damage in lipids and proteins was evaluated. The physical and cardiovascular conditions were evaluated through routine anthropometric parameters (weight, stature and BMI) and blood pressure. Results: Group ( presented significant increases of oxidative stress, reduction in the blood pressure and in the indicators of cardiovascular risks. Group $S$ presented significant reduction of the oxidative stress and increment of the cardiovascular gains. Significance concerning the ergogenic effects has not been identified. Conclusion: Our data suggest that regular exercise in older women can improve physical and cardiovascular conditions. Moreover, daily intake of functional antioxidant supplement can minimize harmful effects of the reactive oxygen species.
\end{abstract}

Keywords: physical activity, antioxidants, oxidative stress, older people, cardiovascular health.

\section{INTRODUÇÃO}

O envelhecimento biológico do ser humano é um fenômeno que está associado a mudanças na atividade das células, tecidos e órgãos, como também com a redução da eficácia de um conjunto de processos fisiológicos ${ }^{(1)}$.

No que tange aos aspectos relativos à produção endógena de oxidantes, tanto as causas como os mecanismos moleculares envolvidos nesse processo ainda não são suficientemente conhecidos. O que se verifica é que as assertivas e hipóteses sobre a influência do processo oxidativo no envelhecimento celular fazem parte de um contexto que envolve, entre outros aspectos, as teorias gerais do envelhecimento. Dentre as várias teorias relativas ao envelhecimento, uma das que tem sido explorada, por conta dos resultados obtidos, é a da restrição calórica. Ou seja, uma das poucas intervenções experimentais que reconhecida- mente tem aumentado o período de vida em animais de laboratório é a denominada "restrição calórica sem desnutrição". As observações em experimentos têm demonstrado que indivíduos submetidos a uma dieta reduzida vivem mais que aqueles que tem acesso livre à alimentação(2). Nessa direção, algumas investigações ${ }^{(2,3)}$ têm apresentado hipóteses sobre o funcionamento da restrição calórica no retardamento da velhice. Uma delas é relacionada à diminuição do processamento da glicose para a produção de ATP. Tal diminuição faria com que também diminuísse a produção de radicais livres e, como decorrência, os danos provocados por esses agentes pró-oxidantes teriam seu efeito retardado. O que se observa é que, embora as investigações ainda procurem desvendar como as mudanças fisiológicas e bioquímicas induzidas pela restrição calórica influem no retardamento do envelhecimento dos mamíferos, as evidências parecem indicar que as mudanças no metabolismo celular se constituem na base desse mecanismo ${ }^{(3,4)}$. 
Vários autores ${ }^{(2-4)}$ divulgaram teorias baseadas nos danos induzidos pelo estresse oxidativo, o qual é produzido tanto por excesso de radicais livres como por ineficácia dos sistemas de defesa antioxidante ${ }^{(5)}$. Desde então, tem sido coletadas evidências que atribuem o envelhecimento à acumulação paulatina de alterações estruturais e funcionais nas macromoléculas e membranas celulares, causadas pelos efeitos deletérios dos radicais livres e outras espécies reativas de oxigênio $(R O S)^{(3,5,6)}$ os quais se formam espontaneamente na mitocôndria como conseqüência do metabolismo oxidativo. Nessa situação de estresse oxidativo, uma parte do ROS escapa às defesas antioxidantes e reage com macromoléculas como lipídeos celulares, proteínas e DNA mitocondrial e nuclear. Como conseqüência deste ataque oxidativo são diminuídas as reservas endógenas de antioxidantes não enzimáticos, alterando a estrutura de lipídios e proteínas celulares, provocando alterações na função de enzimas, no transporte e na sinalização celular, assim como alterações e mutações no material genético. Todo esse conjunto de alterações nos sistemas antioxidantes da célula e o excesso de ROS são gerados tanto pelo envelhecimento das células como também estão presentes entre os mecanismos etiopatogênicos de mais de cem patologias crônicas freqüentes em pessoas idosas, tais como enfermidades cardiovasculares, mal de Parkinson, de Alzheimer, esclerose amiotrófica e o câncer ${ }^{(7,8)}$. Enquanto o sedentarismo e a inatividade física podem conduzir a situações de enfermidade, a prática regular de exercício físico é uma das estratégias mais eficazes na prevenção de enfermidades cardiovasculares e um fator importante na prevenção de patologias crônicas próprias do idoso. Mesmo que sua prática se inicie em idade avançada, o exercício físico é importante para a manutenção de um bom estado geral de saúde, podendo reduzir o risco de diabetes, de algumas formas de câncer e de outras patologias crônicas ${ }^{(8-11)}$. Em relação às enfermidades cardiovasculares (ECV), é sabido que a prática regular de exercício físico, além de aumentar a capacidade cardiorrespiratória ${ }^{(12,13)}$ reduz alguns indicadores de risco tais como a pressão arterial, o colesterol total (CT) e LDL (LDL-C), aumenta os níveis de colesterol HDL e melhora a função endotelial|(11-13) . Por outro lado, também está documentado na literatura que o exercício físico estressante, ou seja, quando o esforço supera a capacidade funcional da pessoa que o realiza, aumenta significativamente o consumo e a utilização muscular de oxigênio, incrementando a produção mitocondrial de ROS, o grau de estresse oxidativo e o dano oxidativo às macromoléculas. Tais aspectos são determinados pelo maior consumo de oxigênio, pela menor eficácia da respiração mitocondrial e diminuição das defesas antioxidantes ${ }^{(11,14)}$. Dessa forma, o balanço entre os efeitos benéficos e os potencialmente danosos do exercício pode ser particularmente importante no caso dos idosos. Tal aspecto justifica verificar se as defesas antioxidantes endógenas de idosos fisicamente ativos são suficientes para neutralizar a formação de ROS associada ao envelhecimento e ao exercício, ou se é necessário o aporte regular de antioxidantes na dieta para prevenir ou reduzir a oxidação de macromoléculas e o risco de ECV.

\section{OBJETIVO}

Verificar a influência da suplementação regular e prolongada de vitaminas antioxidantes na dieta de mulheres que praticam exercícios físicos regulares, sobre o estresse oxidativo, sobre indicadores da saúde física e o risco de ECV.

\section{MÉTODOS}

\section{Participantes, Grupos e Protocolo Experimental}

Foram observadas 68 mulheres com idade entre 60 e 80 anos, não institucionalizadas e residentes na cidade de Salamanca (Espanha). As participantes foram selecionadas dentre o universo de 532 inscritos no Programa de Revitalização Geriátrica realizado por investigadores da Universidade de Salamanca. A seleção dos sujeitos foi feita considerando: participação ( $\geq 75 \%$ ) nas atividades do programa, o sexo e a idade, além dos critérios de inclusão e exclusão adotados pelo programa de Revitalização Geriátrica que levavam em conta dados de disponibilidade pessoal dos indivíduos, de características físicas e de enfermidades que impedissem a participação em qualquer das atividades do programa, tais como: hipertensão não controlada, revascularização cardíaca recente, neoplasias diagnosticadas, osteoartrites avançadas de membros inferiores. As participantes foram subdivididas em: Grupo Controle (Grupo C), integrado por 32 mulheres que realizaram um conjunto de atividades físicas programadas e regulares e cuja dieta habitual não foi suplementada com antioxidantes, e Grupo $\mathrm{S}$, com 36 mulheres, que realizaram o mesmo programa de atividades físicas do Grupo C e cuja dieta habitual foi suplementada com uma bebida funcional antioxidante enriquecida com vitaminas $\mathrm{A}, \mathrm{C}$ e E. Todas assinaram termo de consentimento livre e esclarecido e o estudo foi aprovado pelo Comitê Ético da Universidade de Salamanca (30.06.2004). As sessões de atividade física foram em número de 174, durante 58 semanas, com freqüência de três vezes por semana e duração de 50 a 55 minutos cada uma. Cada sessão envolveu alongamentos miofasciais passivos dos principais grupos musculares (posteriores da perna e coxa, anteriores da coxa e pelve, vertebrais, paravertebrais, peitorais, etc.) com duração de 8 a 10 minutos; ativação cardiocirculatória aeróbia por meio de corrida leve com duração de 9 min.; exercícios adaptados de força, potência e resistência com alteres de mão de 1 e 2 Kg com duração de 7 a 10 minutos; atividades de coordenação, agilidade e flexibilidade (14 a 16 min), exercícios respiratórios e de relaxamento (5 a 7 min) e hidratação em dois momentos da sessão (25 minutos após o início e ao final da sessão). A complementação dietética antioxidante do Grupo $S$ foi feita nos momentos de hidratação de cada sessão de atividade física sob a supervisão profissional de fisioterapeuta e nutricionista, e sem supervisão nos demais dias da semana. Foi realizada mediante a ingestão de $330 \mathrm{ml} /$ dia de uma bebida funcional antioxidante, comercializada com o nome de Funciona $^{\mathrm{TM}}$. Os principais componentes do Funciona ${ }^{\mathrm{TM}}$ são: $33,7 \%$ de suco de frutas (laranja, limão, cenoura, maracujá, abacaxi e acerola), $10 \%$ de leite desnatado, vitaminas $\mathrm{E}(1,5 \mathrm{mg} / 100 \mathrm{~g}, \mathrm{~d}$ - $\alpha$-tocopherol), C (9 mg/100 g, ácido ascórbico) e A (120 g/100 g, ß-caroteno), açúcar, dextrose, aroma, estabilizador (pectina) e água. Os valores nutricionais e energéticos, por $100 \mathrm{~g}$ de bebida são: 0,36 g de proteína, 14,2 g de carboidratos, e 0,07 g de gordura; energia 246 KJ (59 Kcal).

\section{Parâmetros e Indicadores Avaliados}

Como indicadores do estresse e dano oxidativo foram determinadas as concentrações plasmáticas de TBARS -como indicadores de peroxidação lipídica-, de grupos carbonilo -como indicadores de oxidação de proteínas-, assim como de GSH, GSSG no sangue total e calculada a relação molar GSH/GSSG. A concentracão de TBARS foi avaliada pelo método descrito por Ohkawa e cols. ${ }^{(14)}$ e a determinação de grupos carbonilo foi feita com a técnica descrita por Levine e cols. ${ }^{(15)}$ Para a determinação da concentração de glutationa total no sangue foram seguidos os procedimentos descritos por Adams e cols. ${ }^{(16)}$, onde o GSH é seqüencialmente oxidado pelo DTNB e reduzido por NADPH em presença de glutationa reductasa. A taxa de formação o 2-nitro-5tiobenzoico é monitorizada a $412 \mathrm{~nm}$ e se compara com uma solução de GSH de concentração conhecida. Para a determinação do GSSG, foi seguido o procedimento descrito por Ivanov e cols. ${ }^{(17)}$ que utiliza uma técnica de HPLC com detecção fluorescente prévia de tiois livres, com trifenilfosfina e conjugação posterior com monobromobimano que detecta quantidades de aminotiois de 2 pmol. As extrações de sangue foram feitas, de ambos os grupos, antes do início do programa de atividade física (Medida 1) e ao final da intervenção (Medida 2), por profissionais sanitários especializados. As determinações de triacilglicerol, glicose, colesterol total e HDL-colesterol, realizadas no sangue total, foram realizadas por laboratório de análises clínicas credenciado e contratado para tal. Também foram avaliados os parâmetros antropo- 
métricos habituais (peso, altura e índice de massa corporal) e a pressão arterial que foi tomada no início da sessão, no braço esquerdo, com o individuo sentado e por meio de esfigmomanômetro e estetoscópio convencionais. O programa estatístico utilizado para análise dos dados foi o SPSS 11.0, com os testes T- Student e ANOVA.

\section{RESULTADOS}

As alterações observadas nos principais parâmetros antropométricos e nos indicadores de risco cardiovascular associados ao perfil lipídico das mulheres que participaram do estudo podem ser visualizados na Tabela 1. É possível observar que o exercício físico não produziu mudanças estatisticamente significativas no peso corporal, no índice de massa corporal, na glicemia, nos valores de colesterol e de triglicérides das mulheres do grupo controle. Por outro lado, a concentração de colesterol HDL aumentou significativamente diminuindo a relação Colesterol Total/ Colesterol HDL, que se traduz como indicador de risco cardíaco. No Grupo S é possível verificar uma potencialização significativa dos efeitos do exercício sobre o colesterol HDL e sobre o índice de risco cardiovascular, na medida em que o HDL aumentou aproximadamente o dobro em relação ao grupo controle e o índice Colesterol Total/Colesterol HDL também diminuiu em quase duas vezes. Os dados de ambos os grupos relativos à pressão arterial (sistólica e diastólica) podem ser observados na Tabela 2. É possível verificar que os valores basais são similares nos dois grupos. No tocante ao efeito do exercício no grupo controle (Medida 2) observa-se valores de pressão arterial sistólica, diastólica e da média significativamente menores que os obtidos na Medida 1. Esse efeito cardiovascular do exercício físico também foi notado nas mulheres do Grupo S, considerando que os valores foram menores do que os verificados no grupo controle.

Em relação aos fatores relacionados ao estresse oxidativo, pode

Tabela 1. Principais características antropométricas e bioquímicas das mulheres dos grupos Controle ( $n=32$ ) e Suplementado ( $n=36)$. Valores médios e desvio padrão, antes de iniciar o estudo (Medida 1) e depois de finalizar o programa de intervenções (Medida 2).

\begin{tabular}{l|c|c|c|c}
\hline \multicolumn{1}{c}{ GRUPO } & \multicolumn{2}{c}{ CONTROLE } & \multicolumn{2}{c}{ SUPLEMENTADO } \\
\hline \multicolumn{1}{c}{ MEDIDA } & MEDIDA 1 & \multicolumn{1}{c}{ MEDIDA 2 } & \multicolumn{1}{c}{ MEDIDA 1 } & MEDIDA 2 \\
\hline Peso (Kg) & $66,8 \pm 0,5$ & $65,9 \pm 0,6$ & $66.5 \pm 0.4$ & $67,2 \pm 0,5$ \\
\hline $\begin{array}{l}\text { Indice de massa } \\
\text { corporal (kg/m²) }\end{array}$ & $29,3 \pm 0,5$ & $28,7 \pm 0.3$ & $29,0 \pm 0,6$ & $28,8 \pm 0,9$ \\
\hline Glicose (mg/dL) & $98 \pm 3$ & $99 \pm 2$ & $96 \pm 2$ & $93 \pm 2$ \\
\hline $\begin{array}{l}\text { Colesterol total } \\
\text { (mg/dL) }\end{array}$ & $229 \pm 6$ & $220 \pm 5$ & $224 \pm 4$ & $213 \pm 7$ \\
\hline $\begin{array}{l}\text { Colesterol HDL } \\
\text { (mg/dL) }\end{array}$ & $43,3 \pm 0,9$ & $47,9 \pm 1,0^{*}$ & $44,1 \pm 1,1$ & $52,6 \pm 0,8^{*} \#$ \\
\hline CO total/ CO-HDL & $4,97 \pm 0,18$ & $4,51 \pm 0,11^{*}$ & $4,85 \pm 0,15$ & $4,11 \pm 0,10^{*} \#$ \\
\hline Triglicérides (mg/dL) & $98 \pm 6$ & $88 \pm 7$ & $96 \pm 5$ & $91 \pm 5$ \\
\hline
\end{tabular}

* significativamente diferente $(p<0.05)$ em relação ao valor basal (MEDIDA 1)

\# significativamente diferente $(p<0,05)$ em relação ao Grupo Controle

Tabela 2. Valores da pressão arterial sistólica (PAS), diastólica (PAD), média (PAM) e diferencial (Pdif) dos grupos Controle ( $n=32$ ) e Suplementado $(n=36)$. Os valores estão expressos em média e desvio padrão, em mmHg.

\begin{tabular}{|c|c|c|c|c|}
\hline \multirow{2}{*}{$\begin{array}{l}\text { GRUPO } \\
\text { MEDIDA }\end{array}$} & \multicolumn{2}{|c|}{ CONTROLE } & \multicolumn{2}{|c|}{ SUPLEMENTADO } \\
\hline & MEDIDA 1 & MEDIDA 2 & MEDIDA 1 & MEDIDA 2 \\
\hline PAS & $141 \pm 1$ & $137 \pm 2^{*}$ & $140 \pm 2$ & $135 \pm 2^{*}$ \\
\hline PAD & $78,6 \pm 0,6$ & $74,1 \pm 0,9^{*}$ & $76,8 \pm 0,8$ & $71,3 \pm 0,8^{*} \#$ \\
\hline PAM & $98,6 \pm 1,1$ & $93,4 \pm 0,9^{*}$ & $97,5 \pm 0,8$ & $90,3 \pm 0,7$ * \\
\hline Pdif & $62,8 \pm 0,7$ & $63,2 \pm 0,8$ & $63,3 \pm 0,6$ & $63,0 \pm 0,7$ \\
\hline
\end{tabular}

ser observado na Tabela 3, que os valores da peroxidação lipídica (TBARS) sofrem vários tipos de alterações estatisticamente significativas. A primeira que merece destaque é a ocorrida no Grupo C que se caracteriza por um aumento do valor médio de 2,96 para 3,71 $\mu \mathrm{M}$. A segunda é a que pode ser vista no Grupo $S$ e que se caracteriza por uma diminuição do valor médio de 3,10 para 2,20 $\mu \mathrm{M}$. Além dessas alterações, é importante enfatizar que não houve diferença estatisticamente significativa entre as medidas iniciais de ambos os grupos, no entanto, a diferença entre as medidas finais (medidas 2) apresentaram significância. A oxidação protéica, por sua vez, aumenta significativamente no Grupo C e não significativamente no Grupo S e da mesma maneira que a oxidação lipídica, as diferenças não apresentam significância entre as medidas iniciais, mas apresentam entre as médias finais de ambos os grupos. Em relação ao GSH, o que pode ser notado na Tabela 4 é que ocorrem diminuições significativas tanto em um como em outro grupo. Destaque-se que a diminuição ocorrida no Grupo C foi superior à ocorrida no Grupo S, em valores próximos à significância. Finalmente, no que tange à relação GSH/GSSG nas amostras de sangue total (ver Tabela 5), os valores diminuíram no grupo controle, principalmente como conseqüência do aumento significativo dos níveis de GSSG. Por outro lado, essa redução foi antagonizada no Grupo S, de maneira tal que ao finalizar as intervenções se nota uma diferença significativa $(p<0,001)$ entre os valores da Medida 2, sendo que a relação GSH/GSSG observada no Grupo C apresenta valor de $410 \pm 211$ e do Grupo S de $1143 \pm 375$.

\section{DISCUSSÃO}

Os resultados evidenciam que a prática regular de exercícios físicos de intensidade moderada induz o estresse oxidativo em mulheres

Tabela 3. Médias, desvios padrão e significâncias intra-grupos e inter-grupos da peroxidação lipídica (TBARS, em $\mu \mathrm{M}$ ), e da oxidação protéica (Grupos carbonilo, em $\mu M)$, entre as medidas iniciais e finais dos grupos Controle $(n=32)$ e Suplementado $(n=36)$. Signif. para valores inferiores a 0,05

\begin{tabular}{c|c|c|c|c}
\hline \multirow{2}{*}{ GRUPO } & & MEDIDA 1 & MEDIDA 2 & VALOR - P \\
\hline \multirow{2}{*}{ CONTROLE } & TBARS & $2,96 \pm 0,61$ & $3,71 \pm 0,67$ & $<0,001$ \\
\cline { 2 - 5 } & Grupos carbonilo & $30,71 \pm 4,10$ & $37,25 \pm 2,68$ & $<0,001$ \\
\hline \multirow{2}{*}{ SUPLEMENTADO } & TBARS & $3,10 \pm 0,57$ & $2,20 \pm 0,67$ & $<0,001$ \\
\cline { 2 - 5 } & Grupos carbonilo & $30,50 \pm 4,03$ & $31,72 \pm 2,82$ & 0,546 \\
\hline \multirow{2}{*}{ VALOR - P } & TBARS & 0,749 & $<0,001$ & \\
\cline { 2 - 5 } & Grupos carbonilo & 0,996 & $<0,001$ & \\
\hline
\end{tabular}

Tabela 4. Médias, desvios padrão e significâncias intra-grupos e inter-grupos da glutationa plasmática (GSH, em $\mu \mathrm{M})$, entre as medidas iniciais e finais dos grupos Controle $(n=32)$ e suplementado $(S, n=36)$.

\begin{tabular}{c|c|c|c}
\hline GRUPO & MEDIDA 1 & MEDIDA 2 & VALOR - P \\
\hline CONTROLE & $2,73 \pm 0,69$ & $1,72 \pm 0,49$ & $<0,001$ \\
\hline SUPLEMENTADO & $2,80 \pm 0,84$ & $2,23 \pm 0,89$ & 0,024 \\
\hline VALOR -P & 0,990 & $<0,054$ & \\
\hline
\end{tabular}

Signif. para valores inferiores a 0,05

Tabela 5. Médias, desvios padrão e significância do índice GSH/GSSG entre os grupos Controle e Suplementado, ao final do processo de intervenção.

\begin{tabular}{c|c}
\hline GRUPO & INDICE GSH/GSSG \\
\hline CONTROLE & $410,28 \pm 211,55$ \\
\hline SUPLEMENTADO & $1143,08 \pm 375,29$ \\
\hline VALOR - P & $<0,001$ \\
\hline
\end{tabular}

Sign. para valores inferiores a 0,05 
idosas e que o referido estresse pode ser antagonizado quando a dieta é suplementada diariamente com bebida funcional antioxidante. Em relação ao estresse determinado pelo exercício, os dados vão ao encontro dos descritos por Mecoci et al ${ }^{(3)}$, Polidori et $a^{(4)}$ e Jenkins ${ }^{(18)}$ embora esse último não se refira especificamente a idosos. Em relação à utilização de uma dieta rica em antioxidantes e à suplementação com vitaminas, essa afirmação é concordante com os trabalhos de Schneider ${ }^{(19)}$, Polidori et $\mathrm{al}^{(20)}$, e Polidori(21) sendo que este último aponta que a utilização de micronutrientes antioxidantes na dieta, tais como as vitaminas C e E e o betacaroteno, pode promover a prevenção de doenças relacionadas ao envelhecimento.

Os dados indicam também que os efeitos positivos sobre o sistema cardiovascular (aumento dos níveis de HDL, redução do índice colesterol total/colesterol HDL e redução da PA) podem ser potencializados quando a dieta dos sujeitos é suplementada com bebida funcional antioxidante. Embora a prática de exercício físico seja reconhecida como uma estratégia eficaz na prevenção das ECV(13) , quando é realizada de maneira estressante ou por pessoas destreinadas, gera aumento na produção de ROS induzindo ao estresse oxidativo e à peroxidação lipídica. ${ }^{(20)}$ Tal excesso, por sua vez, pode aumentar a oxidação do colesterol LDL, que é um dos processos chaves no aparecimento de placas ateroscleróticas. ${ }^{(7,22)}$ Na Tabela 3, o que se observa é um aumento significativo da oxidação lipídica no Grupo C e, por outro lado, uma diminuição também significativa no Grupo S e, por subseqüência, uma diferença significante entre os valores finais dos grupos. Polidori ${ }^{(21)}$ refere existir um conjunto de evidências que apontam o papel do estresse oxidativo na aterogenesis, e que estudos epidemiológicos sugerem que baixos níveis de antioxidantes estão associados com o incremento das ECV. Portanto, é possível afirmar que os indivíduos que não tomaram antioxidantes tiveram seus valores de oxidação lipídica aumentados no período em que realizaram os exercícios. Da mesma forma é possível a afirmação de que o suplemento antioxidante utilizado provavelmente influiu na peroxidação lipídica induzida pelo exercício, nas mulheres que o ingeriram diariamente.

As proteínas também são facilmente atacadas por ROS, direta ou indiretamente, por meio de lipoperóxidos, modificando sua estrutura e função, estimulando ou inibindo a atividade das que funcionam como enzimas ou como transportadores de membrana, alterando a homeostase iônica transcelular e com ela os níveis intracelulares de cálcio e potássio ou, ainda, de proteínas que funcionam como receptoras ${ }^{(3)}$. No que tange à oxidação protéica, verifica-se que houve um aumento significativo no Grupo C e um aumento não significativo no Grupo S, fato que também indica possível influência do suplemento em relação a esse tipo de oxidação. Considerando que esse tipo de oxidação prejudica não somente as proteínas da membrana celular, mas também as proteínas enzimáticas e da própria mitocôndria, é importante atentar para os prejuízos potenciais determinados por esse processo nos indivíduos com as características do grupo não suplementado.

Outra forma de verificar a ocorrência de condições de estresse oxidativo é pela medição dos níveis de GSH e de GSSG. Em relação a esses indicadores, os resultados evidenciam que, enquanto os valores iniciais de GSH não apresentaram diferença significativa, os níveis de GSH em ambos os grupos sofreram diminuição significativa durante o programa de exercícios (Tabela 4). No entanto, a diminuição no Grupo C apresenta valores mais importantes do que a ocorrida no Grupo S, sendo que a diferença entre os valores finais dos grupos se aproxima da significância estatística, apresentando o valor de $p=0,054$. Dessa forma, é possível verificar que esses dados são concordantes com os de outros trabalhos ${ }^{(5,22)}$ que identificam diminuição do GSH plasmático com a idade. O efeito positivo do suplemento antioxidante foi corroborado pelos índices GSH/GSSG tomados de ambos os grupos, sendo que os cálculos desse índice demonstraram redução significativa no Grupo S em relação ao Grupo C, fato que indica maior proteção contra os efeitos das ROS no grupo S, provavelmente determinada pelos antioxidantes.

\section{CONCLUSÃO}

Em conclusão, os resultados apresentados neste estudo mostram evidências de que uma intervenção nutricional regular e prolongada, por meio de alimentos funcionais antioxidantes, pode ser um agente que previne o estresse oxidativo induzido pelo exercício físico e que provavelmente influi na redução do risco de ECV em mulheres idosas. Nessa direção, considerando as limitações do desenho adotado neste estudo, sugere-se a realização de novos trabalhos com controle de outras variáveis e com a adoção de desenho duplo-cego para análise mais fidedigna de tais evidências.

\section{AGRADECIMENTOS}

Ao Grupo Leche Pascual (Espanha) pelo financiamento parcial deste estudo.

Ao CNPq pela concessão e financiamento da bolsa de pós-doutorado de um dos autores.

Todos os autores declararam não haver qualquer potencial conflito de interesses referente a este artigo.

\section{REFERÊNCIAS BIBLIOGRÁFICAS}

1. Lane MA, Ingram DK, Roth GS. The serious search for an anti-aging pill. Sci Am 2002; 287: 36-41.

2. Lane MA, Ingram DK, Roth GS. Calorie restriction in nonhuman primates: effects on diabetes and cardiovascular risk. Toxicol Sci 1999; 52: 41-8.

3. Mecocci P, Fano G, Fulle S. Age-dependent increases in oxidative damage to DNA, lipids, and proteins in human skeletal muscle. Free Radic Biol Med 1999; 26: 303-8.

4. Polidori MC, Cherubini A, Senin U. Physical activity and oxidation stress during aging. Int I Sports Med 2000; 21: 154-7.

5. McArdle A, Vasilaki A, Jackson M. Exercise and skeletal muscle ageing: cellular and molecular mechanisms. Ageing Res Rev 2002; 1: 79-93.

6. Harman D. Aging: overview. Ann NY Acad Sci 2001; 928: 1-21.

7. Antoniades $C$, Tousoulis D, Tentolouris $C$, Toutouzas $P$, Stefanadis C. Oxidative stress, antioxidant vitamins, and atherosclerosis. From basic research to clinical practice. Herz 2003; 28: 628-38.

8. American College of Sports Medicine, Physical activity programs and behavior counseling in older adult populations. Med Sci Sports Exerc 2004; 36: 1997-2003.

9. Houde SC, Melillo KD. Cardiovascular health and physical activity in older adults: an integrative review of research methodology and results. J Adv Nurs 2002; 38: 219-34.

10. Oguma Y, Sesso HD, Paffenbarger RS Jr, Lee IM. Physical activity and all cause mortality in women: a review of the evidence. Br J Sports Med 2002; 36: 162-72.

11. Mazzeo RS, Cavanagh P, Evans WJ, Fiatarone M, Hagberg J, McAuley E et al. ACSM position stand: exercise and physical activity for older adults. Med Sci Sports Exerc 1998; 30: 992-1008.
12. Halbert JA, Silagy CA, Finucane P, Withers RT, Hamdorf PA. Exercise training and blood lipids in hyperlipidemic and normolipidemic adults: a meta-analysis of randomized, controlled trials. Eur J Clin Nutr 1999; 53: 514-22

13. Hawkins S, Wiswell R. Rate and mechanism of maximal oxygen consumption decline with aging: implications for exercise training. Sports Med 2003; 33: 877-88.

14. Ohkawa H, Ohnishi N, Yagi K. Assay for lipid peroxides in animal tissues by thiobarbituric acid reaction. Anal Biochem 1979; 95: 351-8.

15. Levine RL, Garland D, Olivier N. Determination of carbonyl content of oxidatively modified proteins. Methods Enzymol 1990; 186: 464-7.

16. Adams JD, Lautemburg BH, Mitchel JR. Plasma glutathione and glutathione disulfide in the rat: regulation and response to oxidative stress. J Pharm Exp Ther 1983; 227: 749-54.

17. Ivanov AR, Nazimov IV, Baratova L. Detemination of biologically active low-molecular-mass thils in human blood. J Chromat 2000; 895: 157-66.

18. Jenkins RR. Exercise and oxidative stress methodology: a critique. Am J Clin Nutr 2000; 72: 670-4.

19. Schneider CD, Oliveira AR. Radicais livres de oxigênio e exercício: mecanismos de formação e adaptação ao treinamento físico. Rev Bras Med Esporte 2004; 10: 308-13.

20. Polidori MC, Cherubini A, Senin U, Mecocci P. Peripheral non-enzimatic antioxidant changes with human aging: a selective status report. Biogerontology 2001:2:99-104.

21. Polidori MC. Antioxidant micronutrients in the prevention of age-related diseases. J Postgrad Med 2003; 49: 229-35.

22. Gackowski D, Kruszewski M, Jawien A, Ciecierski M, Olinski R. Further evidence that oxidative stress may be a risk factor responsible for the development of atherosclerosis. Free Radic Biol Med 2001; 31: $542-7$. 